\title{
Analysis of frequency control of multi fans in power station
}

\author{
Zhihang Zhou \\ School of Electrical \& Electronic Engineering, North China Electric Power University Baoding, Baoding \\ 071000, China \\ zhihangzhou@163.com
}

Keywords: fan, frequency control, global dynamic optimization, energy saving.

\begin{abstract}
In this paper, a method of optimizing frequency conversion of fans in power plants based on multi-fan series-parallel mode is proposed. By introducing the theory of global dynamic optimization, this paper sets up the adjustment scheme of the multi fans and tries to use implicit enumeration to solve the problem. It has analyzed the principle of frequency conversion technology, and discussed the optimization by using series parallel power fan theory of regulation. Furthermore, it is of great significance to improve the fan efficiency, save energy, reduce emission, and optimize the industrial structure.
\end{abstract}

\section{Introduction}

"Rich in coal, lack of oil" is China's basic distribution of resources, and this situation has made China's coal-based energy production pattern difficult to change. As of the end of 2010, the national power generation equipment installed capacity is 9.664100 million $\mathrm{kW}$. The thermal power is 7.09670 million $\mathrm{kW}$, accounting for about $73.43 \%$ of the total capacity.

The fan can be energy efficient in the following three aspects ${ }^{[1]}$ : Research and development of energy efficient fan; Reasonable Selection and Reasonable Match of Pipeline; reasonable way to adjust. Among them, the pipeline and efficient fan reasonable match have been basically achieved.

However, more and more peak shaving tasks make the thermal-power-generating-units fans encounter bottlenecks in improving operating efficiency under variable operating conditions. At the same time, more and more researches have been done on reasonable fan regulation methods. In recent years, the rapid development of power electronics technology has been making an important breakthrough in the frequency control speed. The frequency conversion technology is now moving towards high-performance, high-power stage.

This paper makes a deep discussion on the current status of fan frequency control. The energy-saving principles of variable frequency are analyzed firstly, then multi fans frequency control optimization model is established and deeply discussed in further cases based on the the overall dynamic optimization theory, at the end of the paper the conclusion and prospect are put forward finally.

Therefore, it is of great significance to improve the fan efficiency, save energy, reduce emission, and optimize the industrial structure.

\section{The principle of frequency control}

\subsection{Speed calculation}

In a wide range of frequency inverters, VVVF Inverter accounts for a major share of induction ac motor.

Through the induction of asynchronous motor principle, the relationship between the magnetic pole number $\mathrm{P}$, the power frequency $\mathrm{f}$, and $\mathrm{AC}$ induction motor speed $\mathrm{n}_{0 \mathrm{0}}$ an be expressed as:

$$
\mathrm{n}_{0}=\frac{60 * \mathrm{f}}{\mathrm{p}}
$$

Slip ratio of the asynchronous motor: 


$$
\mathrm{S}=\frac{\mathrm{n}_{0}-n}{n_{0}}=1-\frac{n}{n_{0}}
$$

Therefore, the asynchronous motor speed is:

$$
\mathrm{n}=\mathrm{n}_{0}(1-S)=(1-S) 60 * \frac{f_{1}}{p}
$$

\subsection{Energy saving principle}

By the similarity law, the relationship between variable-speed fan flow (Q), power(P), pressure(p) and speed(n) is expressed as the following ${ }^{[2]}$ :

$$
\begin{aligned}
& \frac{\mathrm{Q}_{1}}{Q_{2}}=\frac{n_{1}}{n_{2}} \\
& \frac{\mathrm{p}_{1}}{\mathrm{p}_{2}}=\left(\frac{n_{1}}{n_{2}}\right)^{2} \\
& \frac{\mathrm{P}_{1}}{P_{2}}=\left(\frac{n_{1}}{n_{2}}\right)^{3}
\end{aligned}
$$

It's not difficult to see from (4), (5), (6): Flow is directly proportional to the speed; pressure is proportional to the square of the speed; and power is proportional to the cube of the speed.

Therefore, when the peak shifts, the power can be reduced to three times through the adjust of frequency conversion technology. It is the above-mentioned frequency control advantages which make the current inverter technology in power plant fan applications become more widely.

\section{Multi-fan frequency control optimization}

To ensure adequate safety and reliability of the situation, the current power plants often use centrifugal fan, moving vane adjustable axial fan and static vane adjustable axial fan ${ }^{[3]}$.

Although the frequency control has such advantages, however, the cost of the converter with the large capacity unit supporting is often quite high.So in practice, the power plants often installs variable frequency equipment in some units.

\subsection{Determination of basic parameters of fan}

In the production practice, the fan power consumption, flow, rotational speed, head and other parameters have a certain functional relationship. But because of the complexity of the fan system, we still can not know the relationship between them.

In the performance curve provided by the manufacturer and the test data obtained we can use the neural network to explore their relationship,and we can slso use the least square method for continuous fitting.

So the relationship between pressure and flow can be expressed as:

$$
\mathrm{p}=\mathrm{f}\left(\mathrm{Q}_{\mathrm{i}}\right)
$$

Efficiency and flow:

$$
\eta=g\left(Q_{i}\right)
$$

\subsection{Frequency control mode optimization}

The adjustment of the fan mainly changes with the load of the host group. If the relative change does not match the corresponding transformation, it will cause a huge waste of energy. In this paper, the operation mode of serial-parallel connection is studied under the frequency conversion technology.

\subsubsection{Parallel regulation optimization model}

In the actual production, in order to achieve a specific purpose, the fan is often used in parallel:

1) The need for large flow(but the cost of large flow fan is high )

2) To avoid an accident causing the overall operation to stop

3) Easy to increase or decrease the number of units to adapt to changes in external load requirements 
The fans flow in the same pressure (head) is equal to the sum of all fans when fans are in parallel operation.

At present, the use of fan is often used more than one.So the pursuit of the overall efficiency of the best rather than the optimal operation of a single fan is also of practical significance. In the global dynamic programming theory, the equivalent cost $\mathrm{Z}$ is selected as the objective function (fans have a total of $Y$, including the installation of frequency conversion device $\mathrm{X}$ ):

$$
\min Z=\sum_{i=1}^{y} w_{i} P_{i}+\mathrm{e}_{\mathrm{i}}
$$

Among them, $\mathrm{Z}$ is the equivalent cost, $\mathrm{A}$ (the value $\{0,1\}$ )is the introduced $0-1$ state variable ( 1 is on behalf of the $\mathrm{i}$-fan work, 0 is on behalf of the $\mathrm{i}$-fan not work), $P_{i}$ is the power of $\mathrm{i}$-fan, $e_{i}$ is the potential cost of fan in use (such as life reduction, the damage of devices).

The constraints in parallel are:

System flow constraints:

$$
\sum_{i=1}^{y} w_{i} Q_{i}=Q
$$

variable-frequency fan constraints:

$$
n_{\min i} \leq n_{i} \leq n_{\operatorname{maxi}} \quad \mathrm{i}=1,2 \ldots \mathrm{x}
$$

Other fans speed:

System pressure constraints:

$$
n_{i}=n_{\operatorname{maxi}} \quad i=x+1 \ldots y
$$

$$
p_{i}=p
$$

Fan power constraints:

$$
\mathrm{P}_{\min } \leq P_{i} \leq P_{\max }
$$

Among them, $\mathrm{p}$ is the set value of the outlet manifold wind pressure.

\subsubsection{Series optimization model}

In the actual production, in order to achieve a specific purpose, the fan is often used in series:

1) Designing and manufacturing of a high-pressure fan is more difficult or more expensive

2) Increasing the head is required to output more flow

The fans pressure (head) in the same flow is equal to the sum of all the heads in series operation.

Therefore, the objective function of optimization is:

$$
\min Z=\sum_{i=1}^{y} w_{i} P_{i}+\mathrm{e}_{\mathrm{i}}
$$

Among them, $\mathrm{Z}$ is the equivalent cost, $\mathrm{A}$ (the value $\{0,1\}$ )is the introduced $0-1$ state variable ( 1 is on behalf of the $\mathrm{i}$-fan work, 0 is on behalf of the $\mathrm{i}$-fan not work), $P_{\mathrm{i}}$ is the power of $\mathrm{i}$-fan, $\mathrm{e}_{\mathrm{i}}$ is the potential cost of fan in use (such as life reduction, the damage of devices).

The constraints in series are:

System pressure constraints:

$$
\sum_{i=1}^{y} w_{i} P_{i}=P
$$

variable-frequency fan constraints:

$$
n_{\min i} \leq n_{i} \leq n_{\max i} \quad \mathrm{i}=1,2 \ldots \mathrm{x}
$$

Other fans speed:

$$
n_{i}=n_{\max i} \quad i=x+1 \ldots y
$$

System flow constraints:

$$
Q_{i}=Q
$$

Fan power constraints:

$$
\mathrm{P}_{\min } \leq P_{i} \leq P_{\max }
$$

Among them, $\mathrm{Q}$ is the required capacity of the corresponding units to meet the operation.

\subsubsection{Solving}

Whether in series optimization model or parallel optimization model, we can use the more complete 0-1 complete enumeration method and the implicit enumeration method to find the optimal solution at present. ${ }^{[4][5]}$ 


\section{Example}

A power plant have 6 G4-73№8D centrifugal fans in parallel operation. Three of them are installed the inverter to implementate variable frequency speed control, and the remaining three are not installed frequency control equipment.

Through the test, the rated speed of the fan is 1450rpm, while the minimum limit speed is 700rpm.

The outlet pressure is $200 \mathrm{P}_{\mathrm{a}}$.

The air volume is $76700 \mathrm{~m}^{3} / \mathrm{h}$

According to the 3.2.1(parallel regulation optimization model), the solution: $x=3, y=6$.

There are 63 state values for six fans, and the vector $\mathrm{W}$ can have 63 values, such as all run: $w=[1,1,1,1,1,1]$.

Calculate repeatedly through the constant step of the implicit enumeration method, and use neural networks on the current speed to revise. (The function relationship should be revised because of the frequency conversion)

Finally, we can draw :

$$
\begin{array}{ccc}
\mathrm{W}_{1}=(0,1,0,1,1,0) & n_{1}=(750,0) & F_{1}=38.89 \mathrm{kw} \\
\mathrm{W}_{2}=(0,1,1,1,0,0) & n_{2}=(1230,1230) & F_{1}=32.83 \mathrm{~kW}
\end{array}
$$

So for the above situation, there are two solutions:

Option 1: Two 1230 rpm speed-control fan and a constant speed fan.

Option 2: a 750rpm speed-control fan and two fixed speed fan.

Because the electricity consumption of Scheme I is slightly smaller than that of Option II, it is advisable to adopt Option I as the final solution.

\section{Conclusion and Prospect}

This paper presents an optimization method of variable frequency speed regulation of power plant fans based on multi-fans series-parallel mode. By introducing the theory of global dynamic optimization, the adjustment scheme of multi-fans model is established, and the method of implicit enumeration is used to solve the problems of improving fan efficiency, energy saving, optimizing industrial structure. At the same time with the continuous progress of science and technology, the future energy-saving fans will reach a new level.

\section{References}

[1]LIU Zuo-liang,WANG Zhi-wei,ZHANG Tai-yan,The application of frequency conversion technology in speed adjustment of draft fans and energy saving analysis, Water Conservancy \& Electric Power Machine, Vol. 28,No. 8,pp.1-3

[2] Mechanical Engineering Handbook Motor Engineering Handbook Editorial Board, Mechanical Engineering Handbook, second ed ,1997.8

[3] He Chuan, and Guo Lijun, Pumps and fans.Beijing,2008

[4] L.R. Fulzer, Combinatorial Optimization,Shanghai,1989

[5] Guo B R. Application of optimization technology in thermal power engineering, Beijing 1986 